\title{
Genetic improvement of Purslane (Portulaca oleracea L.) and its future prospects
}

\begin{abstract}
Common purslane (Portulaca oleracea), also known as pigweed, fatweed, pusle, and little hogweed, is an annual succulent herb in the family Portulacaceae that is found in most corners of the globe. From the ancient ages purslane has been treated as a major weed of vegetables as well as other crops. However, worldwide researchers and nutritionists have studied this plant as a potential vegetable crop for humans as well as animals. Purslane is a nutritious vegetable with high antioxidant properties and recently has been recognized as the richest source of Ŭ-linolenic acid, essential omega-3 and 6 fatty acids, ascorbic acid, glutathione, Ŭtocopherol and 6 -carotene. The lack of vegetable sources of $\gamma-3$ fatty acids has resulted in a growing level of attention to introduce purslane as a new cultivated vegetable. In the rapid-revolutionizing worldwide atmosphere, the ability to produce improved planting material appropriate to diverse and varying rising conditions is a supreme precedence. Though various published reports on morphological, physiological, nutritional and medicinal aspects of purslane are available, research on the genetic improvement of this promising vegetable crop are scant. Now it is necessary to conduct research for the genetic improvement of this plant. Genetic improvement of purslane is also a real scientific challenge. Scientific modernization of conventional breeding with the advent of advance biotechnological and molecular approaches such as tissue culture, protoplast fusion, genetic transformation, somatic hybridization, marker-assisted selection, qualitative trait locus mapping, genomics, informatics and various statistical representation have opened up new opportunities of revising the relationship between genetic diversity, agronomic performance and response to breeding for varietal improvement. This review is an attempt to amalgamate the assorted scientific information on purslane propagation, cultivation, varietal improvement, nutrient analyses, medicinal uses and to describe prospective research especially for genetic improvement of this crop.
\end{abstract}

Keyword: Genetic improvement; Portulaca oleracea; Purslane 